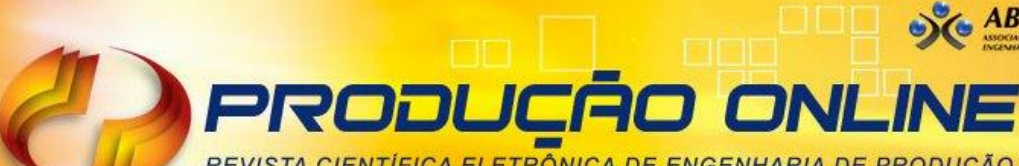 REVISTA CIENTIFICA ELETRÔNICA DE ENGENHARIA DE PRODUÇÃO ISSN 1676-1901
}

\section{UMA ANÁLISE DA EFICIÊNCIA DE PORTOS EUROPEUS POR MEIO DA FERRAMENTA ANÁLISE ENVOLTÓRIA DE DADOS}

\section{AN ANALYSIS OF THE EFFICIENCY OF EUROPEAN PORTS USING THE DATA ENVELOPMENT ANALYSIS TOOL}

\author{
Alvaro Poli Lourenço *E-mail: alvaro.pl@puccampinas.edu.br \\ Denise Helena Lombardo Ferreira* E-mail: lombardo@puc-campinas.edu.br \\ Cibele Roberta Sugahara* E-mail: cibelesu@puc-campinas.edu.br \\ *Pontifícia Universidade Católica de Campinas (PUC-Campinas), Campinas, SP
}

Resumo: Os portos possuem cada vez mais uma posição consolidada em virtude da vital importância na cadeia logística e comercial das nações. Após a expressiva concorrência apresentada com o decorrer da globalização trouxe como necessidade a melhora das ferramentas de gestão portuária. Dessa forma, a presente pesquisa pretende mostrar através da ferramenta Análise Envoltória de Dados, indicadores de eficiência de fatores como input/output. Dados os aspectos capacidade média de transporte por navio, número de contêineres transportados, idade média dos navios, número de chegadas ao porto e o tempo médio de atracação, a fim de estabelecer relações entre os 23 portos analisados com relação à fronteira de eficiência. Os resultados mostram uma disparidade significante entre as eficiências portuárias dos portos internacionais entre os modelos Constant Return ScaleCRS e Variable Return Scale-VRS. O top quatro dos portos eficientes em todas as análises foram Alemanha, Espanha, Bélgica e França. No entanto, pode-se perceber que o modelo VSR se mostrou menos eficiente frente ao CRS.

Palavras-chave: Portos internacionais. Fronteira de eficiência. Ranking. Modelo CRS. Modelo VSR.

Abstract: Ports increasingly have a consolidated position due to the vital importance in the logistics and commercial chain of nations. In this regard, after the significant competition presented with the course of globalization, it was necessary to improve the port management devices. Thus, this research intends to study using the Data Envelopment Analysis tool, presenting efficiency indicators for factors considered input and output. The following data were used such as average transport capacity per ship, number of containers transported, average age of ships, number of arrivals at the port and the average docking time, in order to establish relationships among the 23 DMU's analyzed in relation to the efficiency frontier. The results show a significant disparity between the port efficiencies of international ports between the Constant Return Scale-CRS and Variable Return Scale-VRS. The top four most efficient ports in all analyzes were Germany, Spain, Belgium and France. However, it can be seen that the VRS model was less efficient compared to the CRS.

Keywords: International ports. Efficiency frotier. Ranking. Model CRS. Model VRS.

\section{INTRODUÇÃO}

Atualmente a uma parte significativa da população tem ocupado áreas urbanas em zonas costeiras.

Em 2014, 54\% da população mundial vivia em áreas urbanas, com uma projeção de 66\% até 2050 (UNITED NATIONS, 2014). Além disso, metade da 
população mundial vive a $100 \mathrm{~km}$ da costa (UNEP, 2016), fato que implica em uma ampla relação antrópica com o meio e com o ambiente marinho. Neste contexto, destacam-se as atividades portuárias como sendo importantes para o desenvolvimento, comércio e intercomunicação entre nações.

O transporte marítimo tem se destacado desde a época das navegações, sobretudo no momento atual devido à globalização. Como afirmam Kitzmann, Asmus e Wisniewski (2014), a atividade portuária possui uma importância fundamental no âmbito das zonas costeiras em que se insere, pois, ao possibilitar deslocar pessoas ou cargas, o sistema portuário ocupa uma posição de evidência no sistema de transporte.

Para Falcão e Correia (2012), o porto é considerado um elo na cadeia de transporte, a sua principal finalidade é promover o desenvolvimento econômico de uma região por meio do escoamento de pessoas, de bens e de mercadorias. Segundo Gonzalez e Trujillo (2008), os portos ocupam um importante papel na cadeia logística. Nesse sentido, o nível de eficiência portuária impacta a competitividade de um país, consequentemente uma alta eficiência portuária conduz a baixas tarifas de exportações que, por sua vez, favorecem a competitividade dos produtos nacionais em mercados internacionais.

Segundo Wiegmans, Witte e Spit (2015), o transporte por vias navegáveis, principalmente para o interior dos continentes, é importante há vários séculos, tanto para o transporte de carga e para passageiros. No passado, os cavalos eram usados para puxar as barcaças em direção a seus destinos. Mais tarde a industrialização levou à introdução de motores a vapor no transporte consolidando a introdução do transporte tanto ferroviário quanto por vias fluviais e marítimas para o transporte de mercadorias.

Neste contexto, o transporte ferroviário de mercadorias tornou-se um sério concorrente para o transporte de mercadorias por rotas navegáveis, especialmente para grandes fluxos de frete a granel e para instalações de produção industrial. Já o frete fluvial conseguiu manter sua participação no mercado e desenvolver novos horizontes para o transporte de mercadorias. $O$ frete fluvial é de extrema importância para a mobilidade de produtos como, por exemplo, carga a granel seca e volume líquido, esse conjunto representa aproximadamente $80 \%$ de todo o volume transportado na Europa (WIEGMANS; WITTE; SPIT, 2015).

Revista Produção Online. Florianópolis, SC, v. 20, n. 4, p. 1260- 1278, 2020. 
Por conta dessa consolidação, foi possível desenvolver um novo cenário a partir do transporte por meio de embarcações com a utilização de containers, como já exposto no trabalho de Wiegmans, Witte e Spit (2015). Ao longo dos séculos o papel institucional teve responsabilidade por proporcionar novas oportunidades ao se referir a construções de novos canais, eclusas e novas vias navegáveis. Devido a esses desenvolvimentos históricos e institucionais, a posição dos portos interiores em funções de suas cadeias logísticas também mudou. Tais funções como a de logística se desenvolveu entre tráfego, transbordo e produção para aumentar os serviços de valor, eficiência e logística integrada.

Para apoiar o desenvolvimento econômico orientado para o comércio, as autoridades portuárias cada vez mais agem sob pressão para melhorar a eficiência do porto, garantindo que serviços portuários sejam fornecidos em uma base internacionalmente competitiva. Os portos formam um elo vital em toda a cadeia comercial e, consequentemente, a eficiência portuária contribui para a competitividade internacional de uma nação, como afirma Geoffrey, Jose e Hongyu (1999). Portanto, monitorar e comparar diferentes portos em termos de eficiência de desempenho torna-se essencial para a economia do país de muitos países (BAO; JIAN, 2009).

Neste contexto, essa pesquisa busca mostrar por meio da ferramenta Análise Envoltória de Dados, indicadores de eficiência de fatores como input/output.

O artigo está estruturado com a apresentação desta introdução. A segunda seção descreve a metodologia empregada com o intuito de atender ao objetivo da pesquisa. A terceira seção apresenta os dados selecionados, bem como a análise obtida por meio da ferramenta Análise Envoltória de Dados. Por fim, são expostas as considerações finais do estudo realizado.

\section{METODOLOGIA}

\subsection{Análise Envoltória de Dados}

Para verificar a eficiência de portos europeus, objetivo deste estudo, foi feita a aplicação da ferramenta Análise Envoltória de Dados, em inglês Data Envelopment Analysis (DEA). 
A Análise Envoltória de Dados foi inicialmente proposta por Charnes, Cooper e Rhodes (1978) com o intuito de avaliar a eficiência técnica das escolas públicas primárias nos Estados Unidos. Com a difusão do método DEA no mundo, diversas aplicações foram realizadas nos mais diversos setores, como por exemplo, portuários, indústrias, serviços, entre outros (CHARNES, COOPER; RHODES,1978; COOPER et al., 2006; LINS; MEZA, 2000).

Um dos principais conceitos abordado em DEA é a definição de Decision Making Unit (DMU) - também conhecidas por Unidades Tomadoras de Decisão (UTD) - que representam as entidades a serem avaliadas pela ferramenta DEA. As DMU's devem consumir os mesmos inputs para produzir os mesmos outputs (COOPER et al., 2006; SOUSA JÚNIOR, 2013; BERTOLOTO; MELLO, 2011; TISCOSKI, 2016).

O cálculo da eficiência é dado pela razão entre a soma ponderada dos outpus e a soma ponderada dos inputs. Para isto, o modelo DEA atribui pesos aos inputs e aos outputs para cada DMU observada (DMUo). Em seguida, as DMUo's são avaliadas perante o conjunto de DMU's selecionados, formando, assim, a fronteira eficiente de produção. As DMU's eficientes servem de referência para as DMUS's ineficientes - denominadas de benchmarking (CHARNES; COOPER; RHODES, 1978; ACOSTA et al., 2011; BERTOLOTO; MELLO, 2011; RODRIGUES; AQUINO; THOMAZ, 2015).

A formulação matemática desse problema corresponde a um problema de Programação Fracionária. Esse problema pode ser linearizado e transformado em um problema de Programação Linear (ÂNGULO-MEZA et al., 2005) dado pelas Equações (1) a (4).

A eficiência da DMUo em análise é representada por $h_{o}$, na qual para DMUo em análise são atribuídos os pesos $v_{i}$ aos inputs $x_{i 0}$, tal que $i=1, \ldots, n$; e os pesos $u_{j}$ aos outputs $y_{j 0}$, tal que $j=1, \ldots ., m$. Também são atribuídos os pesos $v_{i}$ e $u_{j}$ aos inputs $i$ e outputs $j$ das demais DMU's $k$, tal que $k=1, \ldots, n$ (ÂNGULO-MEZA et al., 2005).

$\operatorname{Max} h_{o}=\sum_{j=1}^{m} u_{j} y_{j o}$

Revista Produção Online. Florianópolis, SC, v. 20, n. 4, p. 1260- 1278, 2020. 
sujeito a

$$
\begin{aligned}
& \sum_{i=1}^{n} v_{i} x_{i o}=1 \\
& \sum_{j=1}^{m} u_{j} y_{j k}-\sum_{i=1}^{n} v_{i} x_{i k} \leq 0, \quad \mathrm{k}=1,2,3, \ldots, \mathrm{n} \\
& u_{j,} v_{i} \geq 0 \quad \forall i, j
\end{aligned}
$$

Já para a orientação output, é desejada a maximização das saídas mantendose constantes as entradas. Para isso, a razão entre as somas ponderadas da orientação input é invertida. Ao realizar essa ação, a eficiência passa a ser um valor maior que 1. Portanto, para que haja uma maximização das saídas, é realizada uma minimização das entradas (SUGUIY, 2017).

Existem basicamente dois modelos clássicos da ferramenta DEA: o modelo Constant Return Scale (CRS), também conhecido como CCR (CHARNES; COOPER; RHODES, 1978), e o modelo Variable Return Scale (VRS) ou BCC (BANKER; CHARNES; COOPER, 1984). O modelo CRS considera retornos constantes de escala, representado acima pelas Equações (1) a (4). Já o modelo VRS pressupõe retornos variáveis e nenhuma proporcionalidade entre entradas e saídas, representado pelas Equações (5) a (8) (GOMES GARCIA; SILVA; FREITAS, 2017).

$\operatorname{Max} h_{o}=\sum_{j=1}^{m} u_{j} y_{j o}-u_{*}$

sujeito a

$$
\sum_{i=1}^{n} v_{i} x_{i o}=1
$$




$$
\begin{aligned}
& \sum_{j=1}^{m} u_{j} y_{j k}-\sum_{i=1}^{n} v_{i} x_{i k} \leq u_{*}, k=1,2,3, \ldots, n \\
& u_{j,} v_{i} \geq 0 \quad \forall i, j
\end{aligned}
$$

No modelo VRS, a eficiência é dada por $h_{o}$ como no modelo CRS, e $u_{*}$ é um fator de escala, quando positivo indica que a $D M U$ em análise pertence a região de retornos decrescentes de escala; se negativo os retornos de escala são crescentes.

Para ambas as orientações (input ou output), a análise do grau de eficiência de cada uma das unidades produtivas está diretamente relacionada aos pesos atribuídos às variáveis de entrada e de saída seguindo os seguintes pressupostos:

- Se os pesos da DMU analisada ao serem aplicados às demais unidades produtivas resultarem em valores inferiores à dela, esta é eficiente;

- Se a DMU for eficiente somente com seus próprios pesos, ela é denominada "eficiente isolada";

- Se a DMU obtém valores menores de eficiência em relação às demais, mesmo com seus próprios pesos, ela é ineficiente;

- Se ela possui valores maiores de eficiência ao serem aplicados os pesos encontrados para as demais DMU's, ela é uma referência e com base nela será realizado o benchmarking (SUGUIY, 2017).

\subsection{Portos internacionais selecionados}

Para a análise foram selecionados alguns portos internacionais do continente europeu segundo a Tabela 1. 
Tabela 1 - Representatividade dos portos dos respectivos países, bem como as suas localizações geográficas

\begin{tabular}{cc}
\hline País & Localização \\
\hline Bélgica & 1 \\
Bulgária & 2 \\
Dinamarca & 3 \\
Alemanha & 4 \\
Estônia & 5 \\
Irlanda & 6 \\
Grécia & 7 \\
Espanha & 8 \\
França & 9 \\
Croácia & 10 \\
Itália & 11 \\
Chipre & 12 \\
Letônia & 13 \\
Lituânia & 14 \\
Malta & 15 \\
Holanda & 16 \\
Polônia & 17 \\
Portugal & 18 \\
Romênia & 19 \\
Eslovênia & 20 \\
Finlândia & 21 \\
Suécia & 22 \\
Reino Unido & 23 \\
\hline
\end{tabular}

Fonte: Elaboração própria (2020)

A Figura 1 destaca as localizações geográficas dos portos europeus listados na Tabela 1.

Figura 1 - Localização geográfica de cada país

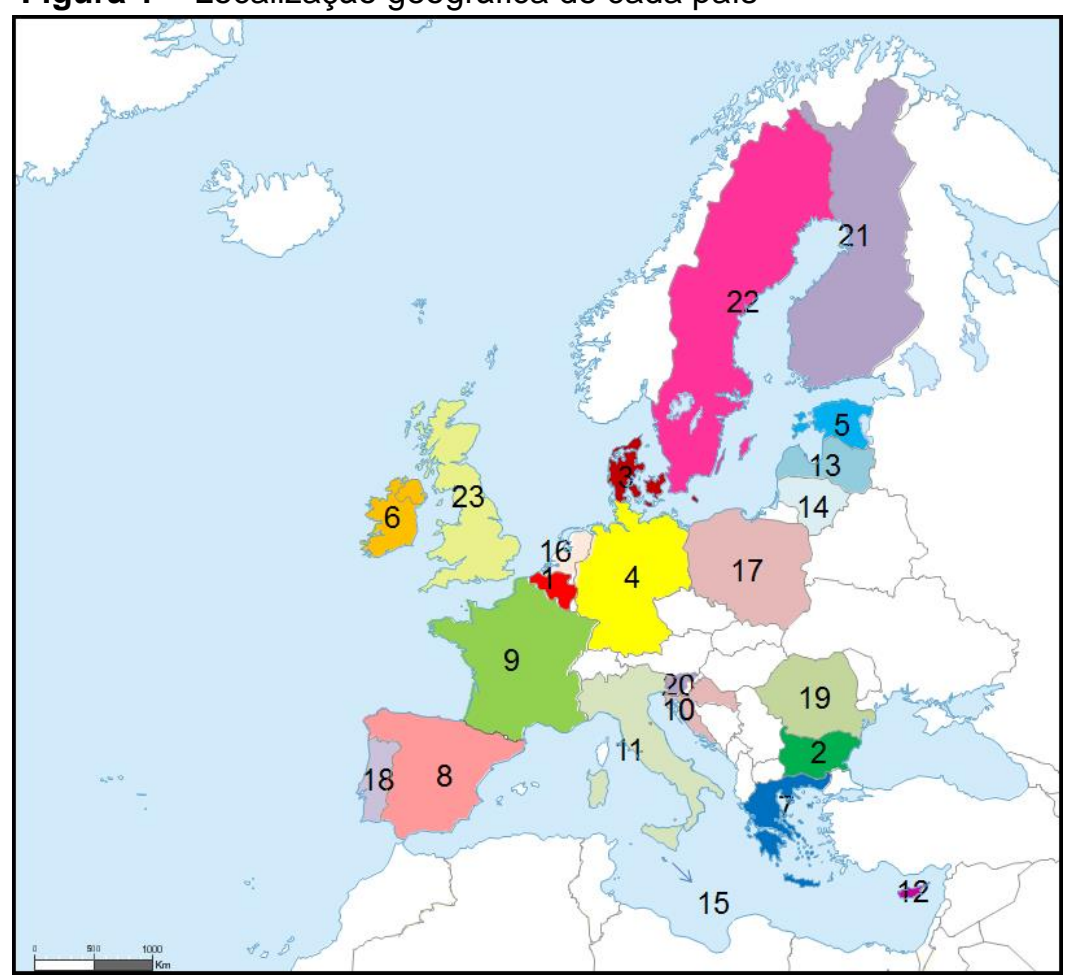

Fonte: Elaboração própria (2020)

Revista Produção Online. Florianópolis, SC, v. 20, n. 4, p. 1260- 1278, 2020. 
É importante destacar que a análise relaciona o conjunto de todos os portos de contêineres de cada país. No entanto, devido ausência de dados, bem como a não compatibilidade temporal entre inputs e outputs levou-se ao dimensionamento dos portos de determinados países componentes da União Europeia. Fato que por meio da base de dados oferecida pela EUROSTAT e UNCTADstat, permitiu que a análise fosse feita no período de 2018 com informações relevantes frente às DMU's.

Neste caso, os dados foram extraídos do banco de dados da United Nations Conference on Trade and Development (UNCTADSstat), por possuírem possíveis informações como inputs e outputs que podem ser trabalhados compartilhando o mesmo período de tempo. Na repartição "Transporte marítimo" encontrou-se a seção de "Indicadores de transportes marítimos" na qual foram obtidos os dados referentes

às "Estatísticas de escala e desempenho dos portos: número de chegadas, tempo gasto nos portos, idade e tamanho do navio, tabela de resumo anual".

\section{APRESENTAÇÃO E ANÁLISE DOS DADOS}

Para a análise foram selecionados alguns portos internacionais do continente europeu Bélgica, Bulgária, Dinamarca, Alemanha, Estônia, Irlanda, Grécia, Espanha, França, Croácia, Itália, Chipre, Letônia, Lituânia, Malta, Holanda, Polônia, Portugal, Romênia, Eslovênia, Finlândia, Suécia e Reino Unido, definidos na Tabela 1.

A ferramenta DEA foi aplicada considerando como DMU's os portos europeus, como inputs: o tempo médio de atracação no porto, a idade média dos navios, e como outputs: a capacidade média do navio, o número de chegadas ao porto e o número de contêineres transportados (Tabela 2). 
Tabela 2 - Dados utilizados para aplicação da DEA, período de 2018

\begin{tabular}{c|c|c|c|c|c}
\hline \multirow{2}{*}{ DMU } & \multicolumn{2}{|c|}{ Inputs } & \multicolumn{3}{c}{ Outputs } \\
\cline { 2 - 6 } & $\begin{array}{c}\text { Tempo } \\
\text { médio no } \\
\text { porto (dias) }\end{array}$ & $\begin{array}{c}\text { Idade } \\
\text { média dos } \\
\text { navios }\end{array}$ & $\begin{array}{c}\text { Capacidade } \\
\text { média por } \\
\text { navio (TEU) }\end{array}$ & $\begin{array}{c}\text { Número } \\
\text { de } \\
\text { chegadas }\end{array}$ & $\begin{array}{c}\text { Número de } \\
\text { contêineres } \\
\text { transportados (TEU) }\end{array}$ \\
\hline Bélgica & 1,0 & 12 & 4882 & 5034 & 12682100 \\
Bulgária & 0,9 & 18 & 1375 & 438 & 217200 \\
Dinamarca & 0,5 & 13 & 1942 & 1171 & 1675900 \\
Alemanha & 0,8 & 11 & 3901 & 9589 & 19597633 \\
Estônia & 0,5 & 14 & 1473 & 388 & 222700 \\
Irlanda & 0,9 & 13 & 895 & 1469 & 988000 \\
Grécia & 0,9 & 14 & 3754 & 3807 & 5324000 \\
Espanha & 0,7 & 13 & 3219 & 14915 & 17189759 \\
França & 0,8 & 12 & 5017 & 4640 & 6369200 \\
Croácia & 0,6 & 12 & 3592 & 394 & 264500 \\
Itália & 0,8 & 14 & 3693 & 8920 & 10547112 \\
Chipre & 0,6 & 16 & 1862 & 722 & 308458 \\
Letônia & 0,7 & 13 & 1275 & 563 & 472532 \\
Lituânia & 0,7 & 14 & 1644 & 844 & 750000 \\
Malta & 1,0 & 12 & 4538 & 2031 & 3314500 \\
Holanda & 0,8 & 12 & 2886 & 12309 & 14825967 \\
Polônia & 0,7 & 13 & 2478 & 1791 & 2834400 \\
Portugal & 0,8 & 15 & 2498 & 3480 & 3199200 \\
Romênia & 1,0 & 14 & 2866 & 543 & 678000 \\
Eslovênia & 0,7 & 14 & 3322 & 629 & 988500 \\
Finlândia & 0,8 & 12 & 1209 & 1896 & 1592090 \\
Suécia & 0,6 & 13 & 1504 & 2161 & 1593100 \\
Reino & 0,7 & 13 & 3464 & 8355 & 11695222 \\
Unido & & & & & \\
\hline Fonte: & $13 N C T D$ & & & \\
\hline
\end{tabular}

Fonte: UNCTADstat (2019).

A Tabela 3 apresenta a estatística descritiva dos dados levantados.

Tabela 3 - Estatística descritiva dos parâmetros inputs e outputs

\begin{tabular}{c|c|c|c|c|c}
\hline Estatísticas & $\begin{array}{c}\text { Tempo } \\
\text { médio no } \\
\text { porto (dias) }\end{array}$ & $\begin{array}{c}\text { Idade média } \\
\text { dos navios }\end{array}$ & $\begin{array}{c}\text { Capacidade média de } \\
\text { transporte de } \\
\text { contêineres (TEU) } \\
\text { por navio contêiner }\end{array}$ & $\begin{array}{c}\text { Número de } \\
\text { chegadas }\end{array}$ & $\begin{array}{c}\text { Número de } \\
\text { contêineres } \\
\text { transportados } \\
\text { TEU }\end{array}$ \\
\hline $\begin{array}{c}\text { Valor } \\
\text { máximo } \\
\text { Valor }\end{array}$ & 1,00 & 18,00 & 5017,00 & 14915,00 & 19597633,00 \\
$\begin{array}{c}\text { mínimo } \\
\text { Média }\end{array}$ & 0,50 & 11,00 & 895,00 & 388,00 & 217200,00 \\
$\begin{array}{c}\text { Desvio } \\
\text { padrão }\end{array}$ & 0,76 & 13,35 & 2751,70 & 3743,00 & 5101307,52 \\
\hline
\end{tabular}

Fonte: Elaboração própria (2020).

Revista Produção Online. Florianópolis, SC, v. 20, n. 4, p. 1260- 1278, 2020. 
Vale ressaltar que a aplicação da ferramenta DEA foi feita por meio do programa Microsoft Excel ${ }^{1} \mathrm{com}$ os dados da Tabela 2 uniformizados.

A Tabela 4 destaca os resultados obtidos pelos diferentes modelos da ferramenta DEA por países (DMU's), e a Tabela 5 mostra a estatística descritiva dos resultados das eficiências obtidas para os modelos CRS-O/I, VRS-O e VRS-I.

Observa-se que para os modelos CRS com orientação a input e com orientação a output obtiveram os mesmos resultados.

Tabela 4 - Resultados da aplicação DEA nos diferentes modelos e orientações adotados

\begin{tabular}{|c|c|c|c|c|c|c|c|c|}
\hline \multirow{3}{*}{ DMU } & \multicolumn{8}{|c|}{ Modelos } \\
\hline & \multicolumn{2}{|c|}{ CRS-O/I } & \multicolumn{2}{|c|}{ VRS-O } & \multicolumn{2}{|c|}{ VRS-I } & \multicolumn{2}{|c|}{ SBM-CRS-O/I } \\
\hline & (a) & (b) & (a) & (b) & (a) & (b) & (a) & (b) \\
\hline Bélgica & 1,00 & 1 & 1,00 & 1,00 & 1,00 & 1 & 1,03 & 4 \\
\hline Bulgária & 0,24 & 22 & 0,27 & 21 & 0,67 & 23 & 0,24 & 22 \\
\hline Dinamarca & 0,62 & 12 & 1,00 & 1 & 1,00 & 1 & 0,62 & 12 \\
\hline Alemanha & 1,00 & 1 & 1,00 & 1 & 1,00 & 1 & 1,37 & 2 \\
\hline Estônia & 0,47 & 17 & 0,76 & 13 & 1,00 & 1 & 0,47 & 17 \\
\hline Irlanda & 0,18 & 23 & 0,20 & 23 & 0,86 & 18 & 0,18 & 23 \\
\hline Grécia & 0,67 & 11 & 0,76 & 12 & 0,81 & 20 & 0,67 & 11 \\
\hline Espanha & 1,00 & 1 & 1,00 & 1 & 1,00 & 1 & 1,38 & 1 \\
\hline França & 1,00 & 1 & 1,00 & 1 & 1,00 & 1 & 1,19 & 3 \\
\hline Croácia & 0,95 & 5 & 1,00 & 1 & 1,00 & 1 & 0,95 & 5 \\
\hline Itália & 0,84 & 9 & 0,90 & 10 & 0,88 & 15 & 0,84 & 9 \\
\hline Chipre & 0,49 & 15 & 0,54 & 16 & 0,83 & 19 & 0,49 & 15 \\
\hline Letônia & 0,29 & 20 & 0,30 & 20 & 0,91 & 13 & 0,29 & 20 \\
\hline Lituânia & 0,37 & 19 & 0,38 & 19 & 0,86 & 16 & 0,37 & 19 \\
\hline Malta & 0,90 & 8 & 0,90 & 9 & 0,96 & 10 & 0,90 & 8 \\
\hline Holanda & 0,92 & 7 & 1,00 & 1 & 1,00 & 1 & 0,92 & 7 \\
\hline Polônia & 0,56 & 13 & 0,59 & 14 & 0,91 & 13 & 0,56 & 13 \\
\hline Portugal & 0,52 & 14 & 0,53 & 17 & 0,80 & 21 & 0,52 & 14 \\
\hline Romênia & 0,49 & 16 & 0,57 & 15 & 0,79 & 22 & 0,49 & 16 \\
\hline Eslovênia & 0,76 & 10 & 0,77 & 11 & 0,86 & 17 & 0,76 & 10 \\
\hline Finlândia & 0,26 & 21 & 0,27 & 22 & 0,94 & 12 & 0,26 & 21 \\
\hline Suécia & 0,42 & 18 & 0,49 & 18 & 0,96 & 11 & 0,42 & 18 \\
\hline $\begin{array}{l}\text { Reino } \\
\text { Unido }\end{array}$ & 0,93 & 6 & 0,96 & 8 & 0,97 & 9 & 0,93 & 6 \\
\hline
\end{tabular}

Fonte: Elaboração própria (2020).

Nota: (a) representa os valores de eficiência obtidos pelos seguintes modelos CRS e VRS para as orientações output/input (O/I) e (b) representa o ranking das DMU's analisadas.

O modelo de super eficiência, em inglês Super Ratio ou Super Eficiency Model (SBM) leva em conta as análises dos modelos CRS e VRS com suas 
respectivas orientações input e output. O modelo tem a finalidade de discretizar as DMU's eficientes e não eficientes que possuem valores muito próximos evidenciados nos modelos CRS e VRS. Segundo Charnes et al. (1985), essa discretização ocorre pelo fato do SBM ser um modelo que trata diretamente os excessos e déficits dos inputs e outputs. Além disso, para se efetuar o cálculo da eficiência, o SBM leva em conta as respectivas folgas correspondentes.

O modelo SBM tem como objetivo expressar as eficiências relativas das DMU's analisadas, bem como o ranking a fim de estabelecer, principalmente, um critério de discretização das DMU's eficientes que apresentaram o mesmo valor de eficiência (100\%) nos modelos CRS e VRS. Deve-se ressaltar que no modelo SBM, diferentemente dos modelos CRS e VRS, as eficiências das DMU's podem resultar valores maiores que 1.

Tabela 5 - Estatística descritiva das eficiências obtidas para os diferentes modelos

\begin{tabular}{c|c|c|c}
\hline \multirow{2}{*}{ Estatística } & \multicolumn{3}{|c}{ Modelos } \\
\cline { 2 - 4 } & CRS-O/I & VRS-O & VRS-I \\
\hline Média & 0,6484 & 0,7041 & 0,913 \\
Valor máximo & 1,000 & 1,000 & 1,000 \\
Valor mínimo & 0,179 & 0,1976 & 0,6668 \\
Desvio padrão & 0,282 & 0,2863 & 0,0918 \\
\hline
\end{tabular}

Fonte: Elaboração própria (2020).

Comparando as estatísticas de eficiências representadas pela Tabela 5, é possível evidenciar um aumento gradual nos parâmetros média, valor mínimo e valor máximo ao usar os diferentes modelos. Neste quesito, tem-se um aumento gradual das eficiências de maneira crescente nos seguintes modelos CRS-O/CRS-I, VRS-O e VRS-I. Os modelos CRS-O e CRS-I apresentam as mesmas eficiências e rankings, pois segundo Charnes, Cooper e Rhodes (1978), esses modelos constroem uma superfície linear por partes, não paramétrica, envolvendo os dados. Além disso, esses modelos usam retornos constantes de escala, isto é, qualquer variação nas entradas (inputs) produz variação proporcional nas saídas (outputs).

Desta forma, analisando os valores, para este cenário tem-se um sutil aumento do CRS-O/I para VRS-O, já na transição dos modelos CRS-O/I e VRS-O para VRS-I é notado um drástico aumento, significando que a eficiência calculada de cada DMU se encontra próxima da fronteira de eficiência do processo. O mesmo aumento é visto para o valor mínimo calculado. Concomitantemente, ocorre o Revista Produção Online. Florianópolis, SC, v. 20, n. 4, p. 1260- 1278, 2020. 
inverso para o desvio padrão, resultando em um valor mínimo para o modelo VRS-I, ao se comparar com os demais analisados. Já o valor máximo se mantém constante devido à máxima eficiência ser o mesmo apresentado para as DMU's eficientes em cada modelo.

A Figura 2 mostra a comparação entre as eficiências das DMU's sob análise utilizando o modelo CRS.

É possível observar que os portos da Alemanha, Bélgica, Espanha e França apresentaram 100\% de eficiência para o modelo CRS para ambas orientações (input e output), contudo o porto da Irlanda teve a menor eficiência (18\%) dentre eles.

Figura 2 - Comparação de eficiências entre as DMU's, modelo CRS-O/I

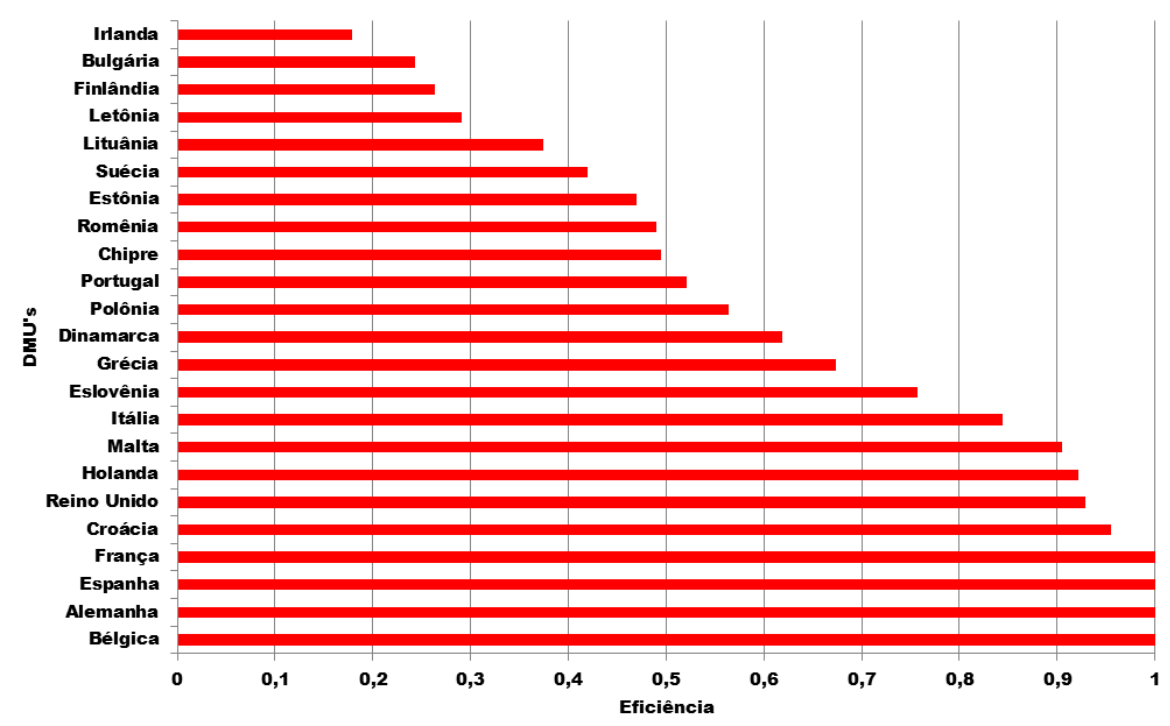

Fonte: Elaboração própria (2020).

A Figura 3 mostra a comparação entre as eficiência das DMU's sob análise utilizando o modelo VRS-O.

Nota-se que os portos pertencentes aos países Alemanha, Bélgica, Croácia, Dinamarca, Espanha, França e Holanda apresentaram 100\% de eficiência para o modelo VRS-O, entretanto o porto da Irlanda teve a menor eficiência (20\%) dentre eles. 
Figura 3 - Comparação de eficiências entre as DMU's, modelo VRS-O

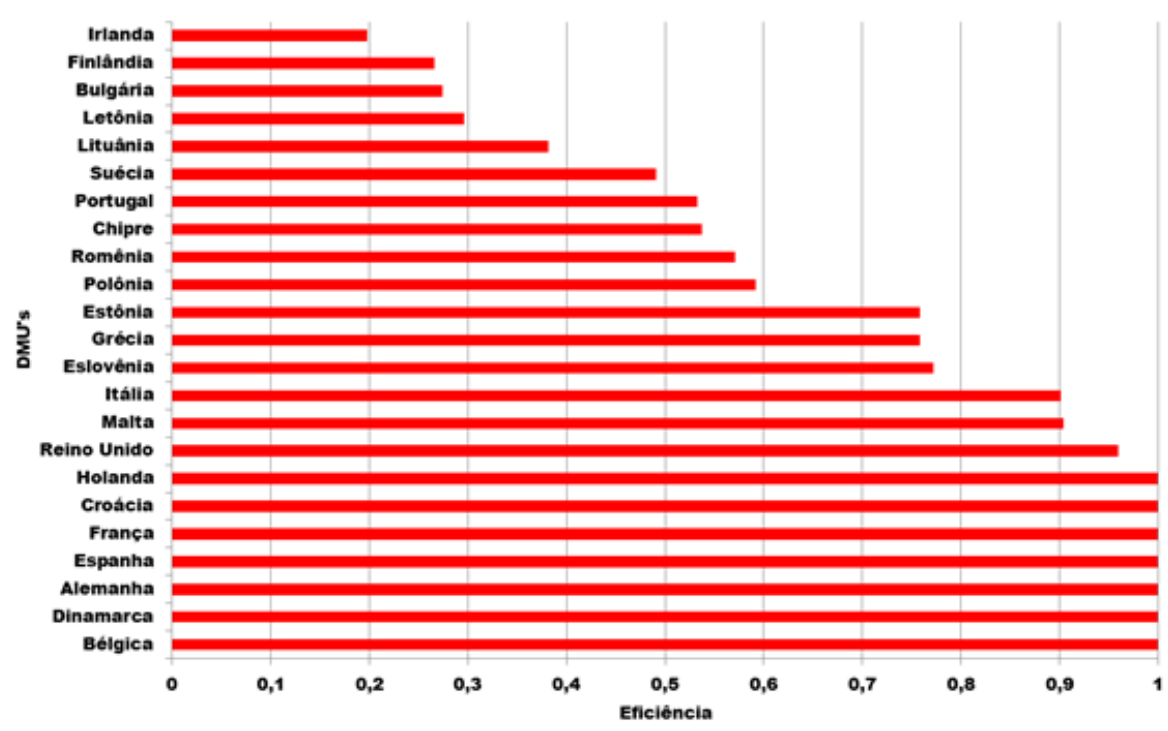

Fonte: Elaboração própria (2020).

A Figura 4 mostra a comparação entre as eficiências das DMU's sob análise utilizando o modelo VRS-I.

Figura 4 - Comparação de eficiências entre as DMU's, modelo VRS-I

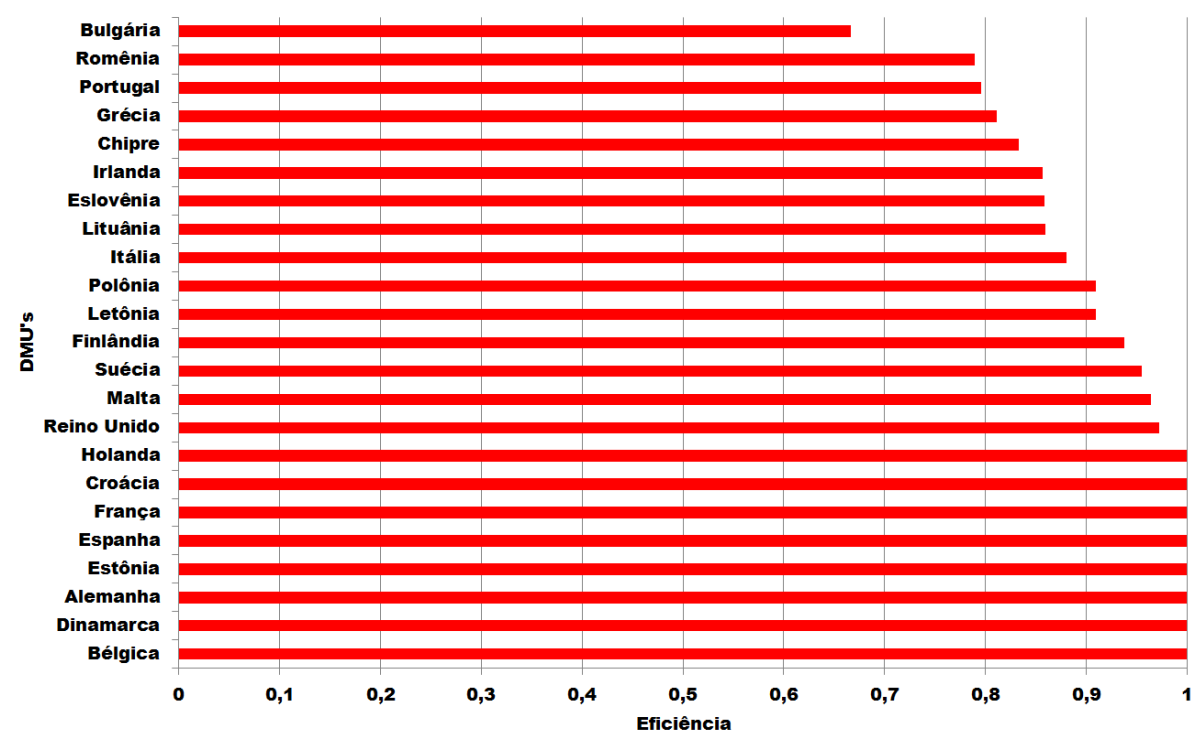

Fonte: Elaboração própria (2020).

Observa-se que os portos pertencentes aos países Alemanha, Bélgica, Croácia, Dinamarca, Espanha, Estônia, França e Holanda apresentaram 100\% de eficiência para o modelo VRS-I, porém o porto da Bulgária teve a menor eficiência 
(67\%) dentre eles. Deve-se destarcar que nesse modelo, as eficências obtidas não foram muito baixas.

A Figura 5 mostra a comparação entre as eficiências das DMU's sob análise utilizando o modelo SBM-CRS-O/I.

Figura 5 - Comparação de eficiências entre as DMU's, modelo SBM-CRS-O e CRS-I

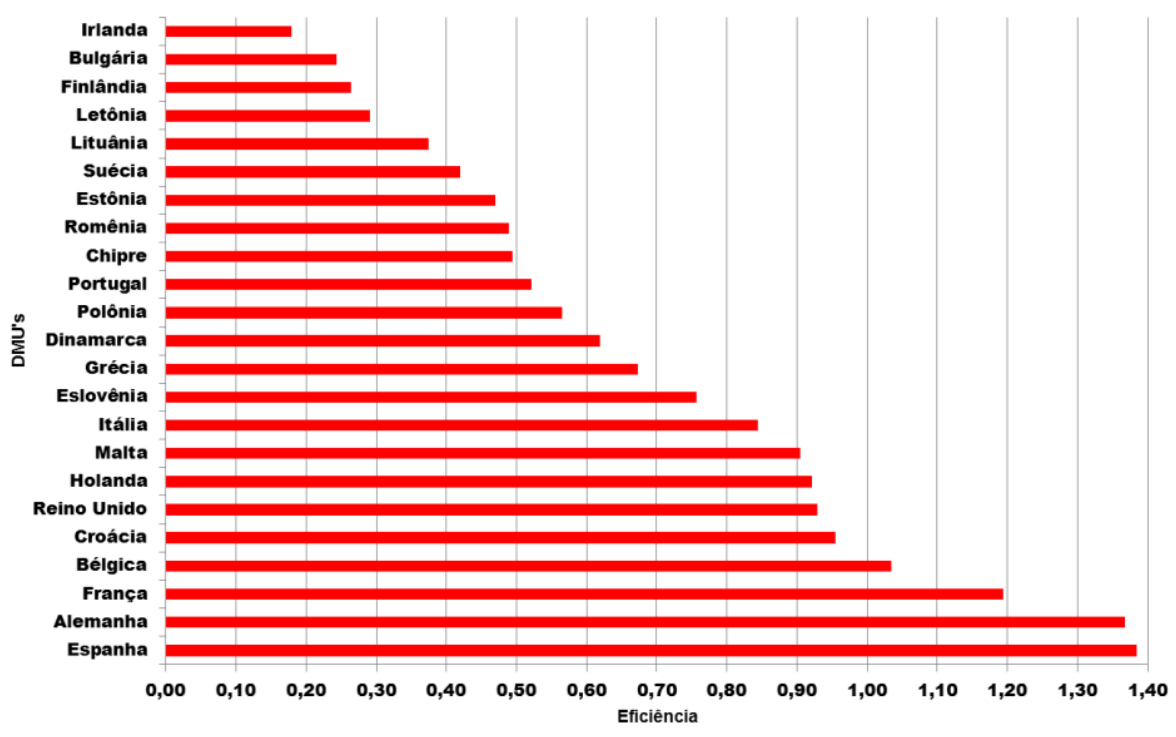

Fonte: Elaboração própria (2020).

É possível observar que o porto da Espanha apresentou maior eficiência (138\%) para o modelo SBM-CRS-O/I, já o porto da Irlanda teve a menor eficiência (18\%) dentre eles.

A eficiência média obtida para os portos analisados nesta pesquisa pode ser comparada com o estudo realizado por Kevin e Teng-Fei (2006), com destaque a similaridade no comportamento entre CRS e VRS. Vale ressaltar que os parâmetros empregados na presente pesquisa se diferenciam aos utilizados por Kevin e TengFei (2006) e como consequência ocorre menor valor da eficiência média. Ademais, os mesmos autores consideram a partição dos portos europeus em áreas sul, oeste e centrais do continente. Para Kevin e Teng-Fei (2006), a eficiência média dos portos de contêineres em diferentes regiões revela que o porto das llhas Britânicas apresenta eficiência média superior às demais regiões, enquanto os terminais de contêineres na Escandinávia e no Leste Europeu têm a menor eficiência média.

Por meio da Tabela 4 e Figuras 2-5, se comparadas entre si, é possível evidenciar por meio dos dados um gradual aumento da eficiência das DMU's entre Revista Produção Online. Florianópolis, SC, v. 20, n. 4, p. 1260- 1278, 2020. 
os modelos CRS-O/I, VRS-O e VRS-I, como visto na análise estatística citada anteriormente.

Além disso, pode-se destacar a mudança no ranking das DMU's, fato que para o modelo CRS-O/I, revelando como os mais expressivos Grécia e Finlândia. Para o porto da Finlândia, os seguintes rankings foram obtidos - 21 (CRS-O/I), 22 (VRS-O) e 12 (VRS-I). Já para o porto da Grécia, resultou-se nos seguintes rankings - 11 (CRS-O/I), 12 (VRS-O) e 20 (VRS-I).

Um estudo similar realizado para os portos asiáticos por Bao e Jian (2009) destacou que o resultado da estimativa de eficiência mostrou que existe uma oportunidade substancial para melhorar a eficiência técnica dos portos marítimos e também a heterogeneidade na eficiência técnica entre portos marítimos do nordeste da Ásia. O resultado revela que três portos marítimos chineses Xangai, Tianjin e Dalian, bem como três portos coreanos Busan, Gwangyang, e Incheon podem ser considerados eficientes dentre os portos analisados presentes no noroeste asiático.

O estudo realizado por Kevin e Teng-Fei (2006) para os portos europeus, destaca que o porto desempenha um papel central na interface entre o transporte marítimo e intermodal terrestre na movimentação de mercadorias e principalmente de contêineres. Uma característica distinta da indústria portuária de contêineres contemporânea é que a concorrência entre portos é cada vez mais intensa. Antigamente o mercado portuário era visto como um monopólio, uma vez que seu caráter exclusio geográfico acarretava um tráfego inevitável de navios ao longo das infraestruturas portuárias. No entanto, o rápido desenvolvimento internacional do transporte intermodal e das estruturas de gestão, ocasiona uma mudança drástica de um monopólio para outro no qual a feroz concorrência é volátil em muitas partes do o mundo.

\section{CONSIDERAÇÕES FINAIS}

Para o modelo CRS tanto orientação output e input resultaram em um mesmo valor de eficiência, ao se analisar os resultados obtidos, pode-se depreender que os valores de inputs retratados como tempo médio no porto (dias) e idade média dos navios têm-se os seus valores mais apropriados quando estão abaixo. Já os valores de outputs, tais como, capacidade média por navio, número de chegadas e número de contêineres transportados, têm-se valores acima da média quando se referem a Revista Produção Online. Florianópolis, SC, v. 20, n. 4, p. 1260- 1278, 2020. 
uma DMU eficiente. Nesse sentido, apenas as DMU's que atingiram a fronteira de eficiência (Bélgica, Alemanha, Espanha e França) evidenciaram este comportamento.

Para o modelo VRS têm-se diferentes resultados tanto à eficiência das DMU's quanto à quantidade que efetivamente chegou à fronteira de eficiência, diante disso a substituição do axioma da proporcionalidade pelo da convexidade é a principal causa dos resultados diferentes ao ser comparados com o modelo CRS. Para o modelo VRS-O, o mesmo pensamento de maximização dos seus ouputs e concomitantemente a minimização dos inputs torna uma DMU eficiente em comparativo com as demais. No entanto, algumas DMU's consideradas eficientes nessa análise possuem alguns valores abaixo da média, sendo o caso da Dinamarca e Croácia, ao se referirem aos seus respectivos outputs. Já para o modelo VRS-I, pode-se notar em comparação ao modelo CRS que possui uma maior quantidade de DMU's consideradas eficientes, dessa maneira os valores dos inputs apresentam de forma maximizada (tal como a Holanda) e bem como alguns outputs apresentam de forma minimizada (caso da Dinamarca, Estônia e Croácia). Nesse quesito, isso propicia que DMU's antes consideradas ineficientes pelo modelo CRS passa a se tornar eficientes por meio da mudança da fronteira de eficiência ocasionada pelo modelo.

Por meio da análise do modelo de super eficiência (SBM), pode-se analisar com maior exatidão o posicionamento das DMU's. Diante disso, DMU's que possuem os mesmos valores de eficiência, torna possível classificar as DMU's presentes na fronteira de eficiência, atribuindo uma pontuação de eficiência maior que 1. Dessa forma, tem-se uma maior margem que se pode abordar a eficiência, mostrando que quanto maior a sua pontuação, mais a DMU é considerada eficiente.

Vale ressaltar que a maior vantagem no quesito de concorrência, estratégica e planejamento na gestão portuária, refere-se à minimização dos recursos de entrada com a maximização dos elementos de saída de um processo. Perante a isso, o modelo CRS-O destaca-se como o mais relevante à análise, bem como a sua orientação no modelo de Super Eficiência ao partir desse pressuposto. Os resultados calculados através do modelo de Super Eficiência, apresentaram uma ordem crescente, s DMU's com maior eficiência foram: Bélgica, França, Alemanha e Espanha. Paralelamente, a falta de precisão calculada para as DMU's da fronteira Revista Produção Online. Florianópolis, SC, v. 20, n. 4, p. 1260- 1278, 2020. 
mostrada no modelo CRS-O deixa de ser um obstáculo nos modelos de Super Eficiência.

Portanto, o modelo CRS aliado com os modelos de Super Eficiência para a análise dos conjuntos de parâmetros analisados como input e output é mais precisa pelo fato de ter DMU's eficientes. Já as análises no modelo VRS, as DMU's que antes eram ineficientes no modelo CRS tornam-se eficientes, além disso, há uma grande variação das DMU's eficientes entre os modelos para as suas diferentes orientações. Dessa forma, nos cálculos de rankings e, sobretudo da eficiência tornam-se totalmente diferentes entre os modelos CRS e VRS, devido ao critério de convexidade da fronteira.

\section{REFERÊNCIAS}

ACOSTA, C. M. M. et al. Aplicação de Análise Envoltória de Dados (DEA) para medir a eficiência em portos brasileiros. Revista de Literatura de Transportes, v. 5, n. 4, p. 88-102, 2011.

ÂNGULO-MEZA, L. et al. ISYDS-Integrated System for Decision Support (SIADSistema Integrado de Apoio à Decisão): a software package for data envelopment analysis model. Pesquisa Operacional, v. 25, n. 3, p. 493-503, 2005. https://doi.org/10.1590/S0101-74382005000300011

BAO J.; JIAN L. DEA-based Performance Measurement of Seaports in Northeast Asia: Radial and Non-radial Approach, The Asian Journal of Shipping and Logistics, v. 25, p. 219-236, 2009. Disponível em: http://www.sciencedirect.com/science/article/pii/S2092521209800035. Acesso em: 27 dez. 2019. https://doi.org/10.1016/S2092-5212(09)80003-5

BANKER, R.D.; CHARNES, A.; COOPER, W.W. Some models for estimating technical scale inefficiencies in data envelopment analysis. Management Science, v. 30, n. 9, p. 1078-1092, 1984. https://doi.org/10.1287/mnsc.30.9.1078

BERTOLOTO, R. F.; MELLO, J. C. C. B. S. de. Eficiência de portos e terminais privados brasileiros com características distintas. Journal of Transport Literature, v. 5 , n. 2, p. 421, 2011.

CHARNES, A. et al. Foundation of data envelopment analyses and ParetoKoopmans empirical production functions. Journal of Econometrics, v. 30, p. 91107, 1985. https://doi.org/10.1016/0304-4076(85)90133-2

CHARNES, A; COOPER, W. W; RHODES, E. Measuring the efficiency of decisionmaking units. European Journal of Operational Research, v. 2, p. 429-444, 1978. https://doi.org/10.1016/0377-2217(78)90138-8 
COOPER, W. W.; SEIFORD, L. M.; TONE, K. Introduction to data envelopment analysis and its use with dea-solver software and references. New York:

Springer Science + Business Media, Inc., 2006.

FALCÃO, V. A.; CORREIA, A. R. Eficiência portuária: análise das principais metodologias para o caso dos portos brasileiros. Journal of Transport Literature, v. 6, n. 4, p. 133-146, 2012. https://doi.org/10.1590/S2238-10312012000400007

GEOFFREY, P.; JOSE, T.; HONGYU, L. Measuring port efficiency: an application of data envelopment analysis, department of economics and statistics, 1999.

GOMES GARCIA, B. T. de; SILVA, M. A. V. da; FREITAS, M. A. V. de. Analysis of Efficiency in the Generation of Waste in General Cargo Port Terminals using DEA. Revista Produção e Desenvolvimento, v. 3, n. 1, p. 60-79, 2017. https://doi.org/10.32358/rpd.2017.v3.203

GONZALEZ, M. M.; TRUJILLO, L. Reforms and infrastructure efficiency in Spain's container ports. Transportation Research Part A, v. 42, p. 243-257, 2008. https://doi.org/10.1016/j.tra.2007.08.006

KEVIN, P. B. C.; TENG-FEI, W. The efficiency of European container ports: a crosssectional data envelopment analysis, International Journal of Logistics Research and Applications, v. 9, n. 1, p. 19-31, 2006. https://doi.org/10.1080/13675560500322417

LINS, M. P. E.; MEZA, L. A. Análise envoltória de dados e perspectivas de integração no ambiente do apoio à decisão. Rio de Janeiro: COPPE/UFRJ, 2000.

KITZMANN, D. I. S.; ASMUS, M. L.; WISNIEWSKI, P. H. Gestão ambiental portuária desafios, possibilidades e inovações em um contexto de globalização. Espaço Aberto, v. 5, n. 2, p. 147-164, 2014. https://doi.org/10.36403/espacoaberto.2014.3308

RODRIGUES, M. V. S.; AQUINO, M. D. de; THOMAZ, A. C. F. Análise da eficiência na produção de energia eólica nos principais estados brasileiros produtores, por meio da aplicação de análise por envoltória de dados. Revista Tecnologia, v. 36, n. 1/2, p. 18-33, 2015. https://doi.org/10.5020/23180730.2015.V36.1/2.18-33

SOUSA JÚNIOR, J. N. C. de. Avaliação da eficiência dos portos utilizando análise envoltória de dados: estudo de caso dos portos da região nordeste do Brasil. Journal of Transport Literature, v. 7, n. 4, p. 75-106, 2013. https://doi.org/10.1590/S2238-10312013000400005

SUGUIY, T. Eficiência versus satisfação no transporte público: um estudo das práticas nas cidades brasileiras. Tese (Doutorado) 160 fls. Programa de PósGraduação em Engenharia Elétrica, Universidade Estadual de Campinas, 2017.

TISCOSKI, J. da S. Análise da eficiência operacional portuária, por meio da análise envoltória de dados: um estudo de caso dos complexos portuários públicos movimentadores de granéis sólidos agrícolas. Dissertação (Mestrado) 105 fls. Programa de Pós-Graduação em Engenharia Civil, Florianópolis-SC, Universidade Federal de Santa Catarina, 2016.

Revista Produção Online. Florianópolis, SC, v. 20, n. 4, p. 1260- 1278, 2020. 
UNCTADstat - United Nations conference on trade and development. New York. Disponível em:

https://unctadstat.unctad.org/wds/TableViewer/tableView.aspx?Reportld=170027. Acesso em: 28 dez. 2019.

UNEP - United Nations Environment Program. Cities and coastal areas, 2016. Disponível em: http://www.unep.org/urban environment/issues/coastal zones.asp. Acesso em: 31 ago. 2019.

UNITED NATIONS. Department of Economic and Social Affairs, Population Division, 2014. World urbanization prospects: The 2014 Revision, Highlights (ST/ESA/SER.A/352).

WIEGMANS, B.; WITTE, P.; Spit T. Inland Port Performance: A Statistical Analysis of Dutch Inland Ports, Transportation Research Procedia, v. 8, p. 145-154, 2015. Disponível em:

http://www.sciencedirect.com/science/article/pii/S235214651500126X. Acesso em: 22 set. 2019. https://doi.org/10.1016/j.trpro.2015.06.050

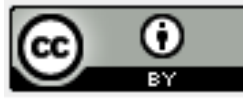

Artigo recebido em: 30/06/2020 e aceito para publicação em: 05/12/2020 DOI: http://dx.doi.org/10.14488/1676-1901. v20i4.4085 